\title{
All binary number-conserving cellular automata based on adjacent cells are intrinsically one-dimensional
}

\author{
Barbara Wolnik \\ Institute of Mathematics, Faculty of Mathematics, Physics and Informatics, University of Gdańsk, 80-308 Gdańsk, Poland \\ Bernard De Baets \\ KERMIT, Department of Data Analysis and Mathematical Modelling, Faculty of Bioscience Engineering, \\ Ghent University, Coupure links 653, B-9000 Gent, Belgium
}

(Received 20 February 2019; revised manuscript received 9 July 2019; published 20 August 2019)

\begin{abstract}
A binary number-conserving cellular automaton is a discrete dynamical system that models the movement of particles in a $d$-dimensional grid. Each cell of the grid is either empty or contains a particle. In subsequent time steps the particles move between the cells, but in one cell there can be at most one particle at a time. In this paper, the von Neumann neighborhood is considered, which means that in each time step a particle can move to an adjacent cell only. It is proven that regardless of the dimension $d$, all of these cellular automata are trivial, as they are intrinsically one-dimensional. Thus, for given $d$, there are only $4 d+1$ binary number-conserving cellular automata with the von Neumann neighborhood: the identity rule and the shift and traffic rules in each of the $2 d$ possible directions.
\end{abstract}

DOI: 10.1103/PhysRevE.100.022126

\section{INTRODUCTION}

When Ulam and von Neumann introduced cellular automata (CAs), they were motivated by biological applications and wanted to create a self-replicating machine, which could be analogous to the human brain and be computationally universal [1,2]. Later, CAs became of interest as models of complex phenomena in various fields of research, such as biology [3], environmental sciences [4], materials science [5], pedestrian dynamics [6], urban transport [7], hydrology [8], and agriculture [9], to name a few. This popularity is due to the fact that CAs reflect the assumption that all laws (physical, economic, sociological, and so on) must result from interactions that are strictly local. In particular, multidimensional CAs seem to be an appropriate tool to model physical phenomena, especially as a model of a system of interacting particles moving in a lattice [10]. A CA interpretation of quantum mechanics based on multidimensional CAs is presented in Ref. [11]: "A cellular automaton is an automaton where the data are imagined to form a discrete, $d$-dimensional lattice, in an $n=d+1$ dimensional space-time." In order to accommodate for conservation laws - one of the most important concepts in physics-usually number-conserving CAs (NCCAs) are used, in which the sum of states is preserved at every update.

In this paper, we study $d$-dimensional NCCAs, where $d$ is a positive integer. From the mathematical point of view, we have a $d$-dimensional rectangular grid consisting of (a possibly huge number of) cells that are capable of storing numerical data; so the cells are assigned states taken from a finite set of possible values. At each consecutive time step, the states of each cell and its neighbors are used by a local rule to compute the new state of the cell and this is applied simultaneously to all cells in the grid. Additionally, we assume that the following three conditions are met: the state set consists only of zero and one (binary CAs), the local rule uses the von Neumann neighborhood, and the grid is finite (with periodic boundary conditions).

Each NCCA can be regarded as a model of a $d$-dimensional interacting particle system. In the context of the movement of particles, the above assumptions have a natural interpretation, which we provide next, illustrating that our assumptions are not too restrictive.

The first assumption means that at each time step every cell is either empty or contains one particle. Note that we usually do not need to consider a model where in one cell there could be more than one particle, because we can assume that the size of the cells is as small as we need (for example, of the order of the Planck scale, i.e., $1.62 \times 10^{-35} \mathrm{~m}$ ). However, two states only usually do not allow to encode the influence of an external force resulting, for example, from the impact of a nonuniform gravitational field.

The second assumption states that at each time step a particle can move to an adjacent cell only, i.e., to a cell having a common $(d-1)$-dimensional facet. This assumption is not very restrictive as we can use a sufficiently small scale of time (of the order of the Planck scale, i.e., $5.39 \times 10^{-44} \mathrm{~s}$ ).

The third assumption stating that the grid is finite and that periodic boundary conditions are used is made only for our convenience. Indeed, Durand et al. [12] considered three types of number conservation (periodic, finite, infinite) and proved their equivalence.

The vast majority of results on binary CAs concerns one-dimensional ones. This is connected with the double exponential increase of the number of possible local rules: If the dimension of the space is $d$, then the number of cells forming the von Neumann neighborhood equals $2 d+1$, and 
thus there are $2^{2^{2 d+1}} \mathrm{CAs}$ in this case. One-dimensional binary CAs, better known as elementary CAs (ECAs), are fairly well researched and, in particular, it is known that among all 256 of them, there are only five that are number conserving: the identity rule (ECA 204), the shift-left rule (ECA 170), the shift-right rule (ECA 240), the traffic-right rule (ECA 184), and the traffic-left rule (ECA 226) [13]. Increasing the dimension by one yields as many as 4294967296 twodimensional binary CAs, yet only four new NCCAs appear: the shift and the traffic rules in the downward and upward direction. If $d=3$, then the cardinality of the set of all CAs increases to the incredibly large number $2^{128} \approx 3.4 \times 10^{38}$, but using the characterization of number-conserving CAs given in Ref. [14], it is still possible to find all NCCAs and we obtain that there are only 13 , namely the identity rule, six shift rules, and six traffic rules (in each of the possible directions: right, left, up, down, forward, and backward).

A new approach to study NCCAs was recently proposed in Ref. [15]. The main result of that paper, the decomposition theorem, states that the local rule of any number-conserving CA can be decomposed into two parts: a split function and a perturbation. This decomposition is unique and, moreover, the set of all split functions has a very simple structure, while the set of all perturbations forms a linear space and is therefore very easy to describe in terms of a basis. This idea allows to reduce the computational complexity of finding all NCCAs for classical combinations of dimension $d$ and state set.

In this paper, we show that the decomposition theorem can also be a convenient tool for proving some general properties concerning $d$-dimensional NCCAs. We confirm the hypothesis that in the binary case, increasing the space dimension does not result in the appearance of a new kind of number-conserving CA (see Ref. [15]), as we get that for a given dimension $d$, there are exactly $4 d+1$ numberconserving binary CAs with the von Neumann neighborhood: the identity rule, and the shift and the traffic rules in each of the $2 d$ possible directions. Thus, all of them are intrinsically one-dimensional. In this way, our result fully unveils the structure of the $d$-dimensional number-conserving binary CAs with the von Neumann neighborhood. Moreover, it is obtained through the use of basic mathematical tools, similarly as the famous result of Land and Belew [16], which fully resolved the problem of existence of a perfect solution of the density classification problem.

\section{PRELIMINARIES}

In this paper, we adhere to the notations introduced in Ref. [15], where also some explanations and examples are given. Here we present only a short description without details.

Let us fix the dimension $d \geqslant 1$ and natural numbers $n_{1}, n_{2}, \ldots, n_{d}$ greater than four. We consider the cellular space as a $d$-dimensional finite grid:

$$
\mathcal{C}=\left\{0,1, \ldots, n_{1}-1\right\} \times \ldots \times\left\{0,1, \ldots, n_{d}-1\right\} .
$$

With this notation, each cell $\mathbf{i} \in \mathcal{C}$ is a $d$-tuple $\left(i_{1}, \ldots, i_{d}\right)$, where $i_{k} \in\left\{0,1, \ldots, n_{k}-1\right\}$. As mentioned in the Introduction, we consider a finite grid only for our convenience, as in

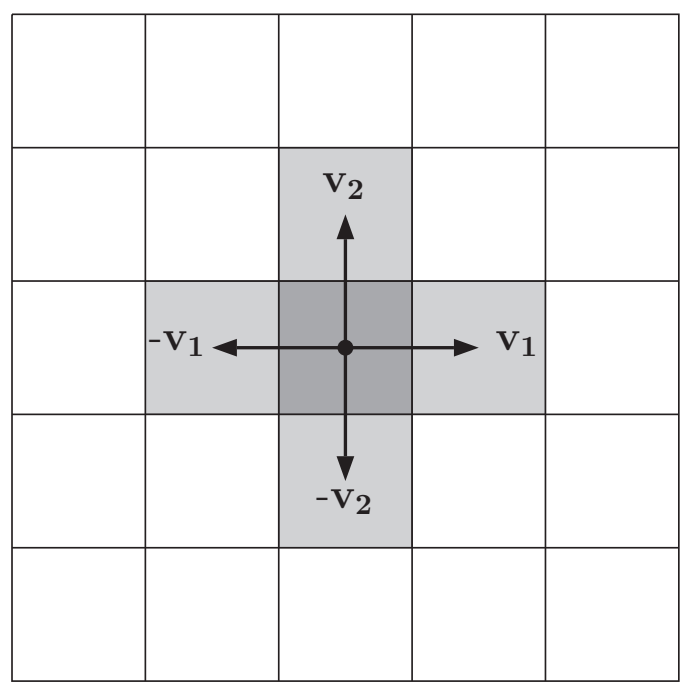

FIG. 1. The von Neumann neighborhood $P(\mathbf{i})$ in the case of $d=$ 2. The cell $\mathbf{i}$ is marked in dark gray and the cells adjacent to it are marked in light gray.

this case there is no problem with the definition of the sum of all states in a configuration. However, according to Durand et al. [12], the results obtained are also valid for infinite grids.

For each $k \in\{1,2, \ldots, d\}$, we define the vector $\mathbf{v}_{k}=$ $(0, \ldots, 0,1,0, \ldots, 0) \in \mathbb{R}^{d}$, where the $k$ th component is 1 and the other ones are zero. Let us denote the set of all directions as $V_{+}$, i.e., $V_{+}=\left\{\mathbf{v}_{1},-\mathbf{v}_{1}, \mathbf{v}_{2},-\mathbf{v}_{2}, \ldots, \mathbf{v}_{d},-\mathbf{v}_{d}\right\}$. For example, if $d=2$, then $V_{+}$consists of four directions: $\mathbf{v}_{1}=(1,0)$ (right), $-\mathbf{v}_{1}=(-1,0)$ (left), $\mathbf{v}_{2}=(0,1)$ (up), and $-\mathbf{v}_{2}=(0,-1)$ (down).

We consider periodic boundary conditions, i.e., the cell adjacent to the cell $\mathbf{i}=\left(i_{1}, \ldots, i_{d}\right)$ in the direction $\mathbf{v}_{k}$ is equal to $\left(i_{1}, \ldots, i_{k-1}, i_{k}+1, i_{k+1}, \ldots, i_{d}\right)$, where the sum is understood modulo $n_{k}$. Moreover, if $\mathbf{j}$ is adjacent to $\mathbf{i}$ in the direction $\mathbf{v}_{k}$, then $\mathbf{i}$ is adjacent to $\mathbf{j}$ in the direction $-\mathbf{v}_{k}$. As a consequence, each cell in $\mathcal{C}$ has exactly $2 d$ adjacent cells: one in each of the directions from $V_{+}$.

Additionally, let $\mathbf{0}=(0,0, \ldots, 0) \in \mathbb{R}^{d}$ and $V=V_{+} \cup$ $\{\mathbf{0}\}$. We use the following notation: For $\mathbf{i} \in \mathcal{C}$ and $\mathbf{v} \in V$, $\mathbf{i}+\mathbf{v} \in \mathcal{C}$ is nothing but the cell adjacent to $\mathbf{i}$ in direction $\mathbf{v}$ if $\mathbf{v} \in V_{+}$or $\mathbf{i}$ itself if $\mathbf{v}=\mathbf{0}$.

For each cell $\mathbf{i} \in \mathcal{C}$, its von Neumann neighborhood $P(\mathbf{i})$ consists of the cell itself and its $2 d$ adjacent cells:

$$
P(\mathbf{i})=\mathbf{i}+V:=\{\mathbf{i}+\mathbf{v} \mid \mathbf{v} \in V\} .
$$

The von Neumann neighborhood in the case of $d=2$ is shown in Fig. 1.

In the remainder of this paper, we consider only CAs with the von Neumann neighborhood, even if we do not emphasize it in some places.

Now, we define $\boldsymbol{\Omega}$ as a set containing all pairs $\{\mathbf{0}, \mathbf{v}\}$, where $\mathbf{v} \in V_{+}$, and all pairs $\{\mathbf{u}, \mathbf{v}\}$ for $\mathbf{u}, \mathbf{v} \in V_{+}$, for which $\mathbf{u} \neq \mathbf{v}$ and $\mathbf{u} \neq-\mathbf{v}$. As the pairs $\{\mathbf{u}, \mathbf{v}\}$ and $\{\mathbf{v}, \mathbf{u}\}$ are equal, the set $\boldsymbol{\Omega}$ contains exactly $2 d^{2}$ elements. For example, if $d=2$, then $\boldsymbol{\Omega}$ contains eight elements:

$$
\begin{aligned}
\boldsymbol{\Omega}= & \left\{\left\{\mathbf{0}, \mathbf{v}_{1}\right\},\left\{\mathbf{0}, \mathbf{v}_{2}\right\},\left\{\mathbf{0},-\mathbf{v}_{1}\right\},\left\{\mathbf{0},-\mathbf{v}_{2}\right\},\right. \\
& \left.\left\{\mathbf{v}_{1}, \mathbf{v}_{2}\right\},\left\{\mathbf{v}_{1},-\mathbf{v}_{2}\right\},\left\{-\mathbf{v}_{1},-\mathbf{v}_{2}\right\},\left\{-\mathbf{v}_{1}, \mathbf{v}_{2}\right\}\right\} .
\end{aligned}
$$


For a given pair $\{\mathbf{u}, \mathbf{v}\} \in \boldsymbol{\Omega}$, the pair $\{-\mathbf{u},-\mathbf{v}\}$ is called the matching pair. If from each two matching pairs, we choose exactly one, then we get a set, which we denote by $\boldsymbol{\Lambda}$. Note that $|\boldsymbol{\Lambda}|=d^{2}$. We can construct $\boldsymbol{\Lambda}$ in $2^{d^{2}}$ ways, for example, we can choose

$$
\boldsymbol{\Lambda}=\bigcup_{i=1}^{d}\left\{\left\{\mathbf{0}, \mathbf{v}_{i}\right\}\right\} \cup \bigcup_{i=1}^{d-1} \bigcup_{j=i+1}^{d}\left\{\left\{\mathbf{v}_{i}, \mathbf{v}_{j}\right\},\left\{-\mathbf{v}_{i}, \mathbf{v}_{j}\right\}\right\}
$$

For example, if $d=2$, then such $\boldsymbol{\Lambda}$ contains four elements:

$$
\boldsymbol{\Lambda}=\left\{\left\{\mathbf{0}, \mathbf{v}_{1}\right\},\left\{\mathbf{0}, \mathbf{v}_{2}\right\},\left\{\mathbf{v}_{1}, \mathbf{v}_{2}\right\},\left\{\mathbf{v}_{1},-\mathbf{v}_{2}\right\}\right\} .
$$

For a binary CA, we consider the state set $Q=\{0,1\}$. By a configuration, we mean any function from the grid $\mathcal{C}$ to $\{0,1\}$ and the set of all configurations is denoted by $X=\{0,1\}^{\mathcal{C}}$. We consider also configurations in a wider sense: mappings from the grid $\mathcal{C}$ to $\mathbb{R}$. The set of all configurations in a wider sense is, of course, a superset of $X$ and is denoted by $\widetilde{X}$. We will simply write configuration also for elements from $\widetilde{X}$, unless confusion is possible. The value of cell $\mathbf{i}$ in a configuration $\mathbf{x} \in \widetilde{X}$ is denoted by $\mathbf{x}(\mathbf{i})$ and we define the sum of the states in $\mathbf{x}$ as $\sigma(\mathbf{x})=\sum_{\mathbf{i} \in \mathcal{C}} \mathbf{x}(\mathbf{i})$.

Let us recall that the neighborhood of each cell is labeled by the elements of the set $V$ [see Eq. (1)]. Thus it is natural to define a neighborhood configuration as a function $N: V \rightarrow$ $\{0,1\}$. The set of all possible neighborhood configurations is denoted by $\mathcal{N}$. In the case of $d=2$, it is helpful to represent a neighborhood configuration $N$ graphically as $\begin{gathered}q_{1} \\ q_{2} \\ q_{3} \\ q_{5}\end{gathered}$ with $N(\mathbf{0})=q_{3}, N\left(\mathbf{v}_{1}\right)=q_{4}, N\left(-\mathbf{v}_{1}\right)=q_{2}, N\left(\mathbf{v}_{2}\right)=q_{1}$, and $N\left(-\mathbf{v}_{2}\right)=q_{5}$. Using this notation, the set $\mathcal{N}$ can be expressed as:

$$
\mathcal{N}=\left\{\begin{array}{ccc} 
& q_{1} & \\
q_{2} & q_{3} & q_{4} \mid q_{1}, q_{2}, q_{3}, q_{4}, q_{5} \in Q \\
& q_{5}
\end{array}\right. \text {. }
$$

Some particular neighborhood configurations will be very important in the remainder of this paper. First, for any $\mathbf{v} \in V$ and $q \in\{0,1\}$, we define a monomer $M_{\mathbf{v}: q}$, which has the value $q$ in direction $\mathbf{v}$ and zero in the other directions, i.e., for $\mathbf{u} \in V$ we put

$$
M_{\mathbf{v}: q}(\mathbf{u})= \begin{cases}q, & \text { if } \mathbf{u}=\mathbf{v} \\ 0, & \text { if } \mathbf{u} \neq \mathbf{v}\end{cases}
$$

Note that if $q=0$, then the neighborhood configuration $M_{\mathbf{v}: q}$ is a zero function, irrespective of $\mathbf{v}$, and we denote it also by $M_{0}$. For example, if $d=2$, then there are five monomers besides $M_{0}$ :

$$
\begin{array}{llllllllllll} 
& 1 & & 0 & & 0 & & 0 & & 0 & \\
0 & 0 & 0,1 & 0 & 0,0 & 1 & 0,0 & 0 & 1,0 & 0 & 0 \\
& 0 & & 0 & & 0 & & 0 & & 1 &
\end{array} .
$$

Second, if $\{\mathbf{u}, \mathbf{w}\} \in \boldsymbol{\Omega}$ and $p, q \in\{0,1\}$, then we introduce a dimer $D_{\substack{\text { u: } p: \\ \text { w.q }}}$ as the neighborhood configuration that has the value $p$ in direction $\mathbf{u}$, the value $q$ in direction $\mathbf{w}$ and zero in the other directions, i.e., for $\mathbf{v} \in V$ we put

$$
D_{\mathbf{w}}^{\mathbf{w}: p} \underset{q}{ }(\mathbf{v})= \begin{cases}p, & \text { if } \mathbf{v}=\mathbf{u} \\ q, & \text { if } \mathbf{v}=\mathbf{w} \\ 0, & \text { otherwise }\end{cases}
$$

For example, if $d=2$, then the dimers are of the form:

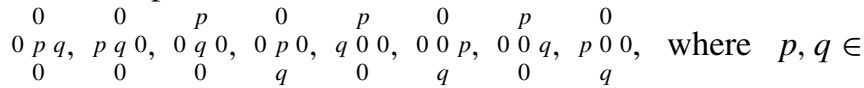
$Q$. Note that the states $p$ and $q$ occur at specific locations as determined by the set $\boldsymbol{\Omega}$.

Of course, $D_{\mathbf{w}: p}$ equals $D_{\mathbf{w}}: q$, and if $p=0$ or $q=0$, then $D_{\mathbf{w}}: p$ is a monomer. As the pairs $\{\mathbf{u}, \mathbf{w}\}$ and $\{-\mathbf{u},-\mathbf{w}\}$ are called matching, we also use this term to refer to the dimers

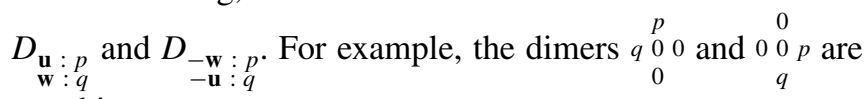
matching.

If $\mathbf{x} \in X$ and $\mathbf{i} \in \mathcal{C}$ are given, then $N_{\mathbf{x}, \mathbf{i}}$ denotes the neighborhood configuration given by the von Neumann neighborhood of the cell $\mathbf{i}$ in the configuration $\mathbf{x}$, thus for $\mathbf{v} \in V$ we have $N_{\mathbf{x}, \mathbf{i}}(\mathbf{v})=\mathbf{x}(\mathbf{i}+\mathbf{v})$.

Any function $f: \mathcal{N} \rightarrow \mathbb{R}$ is called a local function. If additionally $f(\mathcal{N}) \subseteq\{0,1\}$, then we call $f$ a local rule. A local function $f$ induces a global function $A_{f}: X \rightarrow \widetilde{X}$ defined for $\mathbf{x} \in X$ and $\mathbf{i} \in \mathcal{C}$ as follows:

$$
A_{f}(\mathbf{x})(\mathbf{i})=f\left(N_{\mathbf{x}, \mathbf{i}}\right) .
$$

If $f$ is a local rule, then we call $A_{f}$ a global rule and then $A_{f}$ : $X \rightarrow X$. If a global function $A_{f}$ conserves the sum of states, i.e., for each $\mathbf{x} \in X$ it holds that $\sigma\left(A_{f}(\mathbf{x})\right)=\sigma(\mathbf{x})$, then the local function $f$ is called number conserving.

In the one-dimensional case, considering the von Neumann neighborhood leads to 256 ECAs. Among them, as we mentioned in the introduction, there are only five numberconserving ones. We present these rules by means of their look-up table in Table I. The identity rule and the shift rules are stationary: The pattern of a configuration does not change; it can only move to the right or to the left. More interesting are the traffic rules, which are often used as the simplest model of road traffic flow. The states 1 and 0 are then interpreted as "car" and "empty space," respectively. In such model, cars are moving only if there is an empty space in front of them. The dynamics of these rules is well understood [17].

It turns out that any number-conserving local rule can be decomposed into two easy-to-describe parts: a split function and a perturbation. Both of these objects were introduced in Ref. [15]. The theory presented there is developed for the general case of an arbitrary state set $Q \subseteq \mathbb{R}$. In this paper, we consider the simplest state set, namely $Q=\{0,1\}$, and for this reason the definitions and facts from Ref. [15] can be reformulated in a simpler way. We start with the definition of a split function.

TABLE I. The only ECAs that are number conserving.

\begin{tabular}{lcccccccc}
\hline \hline & 111 & 110 & 101 & 100 & 011 & 010 & 001 & 000 \\
\hline Identity rule & 1 & 1 & 0 & 0 & 1 & 1 & 0 & 0 \\
Shift-left rule & 1 & 1 & 1 & 1 & 0 & 0 & 0 & 0 \\
Shift-right rule & 1 & 0 & 1 & 0 & 1 & 0 & 1 & 0 \\
Traffic-right rule & 1 & 0 & 1 & 1 & 1 & 0 & 0 & 0 \\
Traffic-left rule & 1 & 1 & 1 & 0 & 0 & 0 & 1 & 0 \\
\hline \hline
\end{tabular}


Definition II.1. For a given $\mathbf{w} \in V$, let $h_{\mathbf{w}}$ be the local function defined on monomers by

$$
h_{\mathbf{w}}\left(M_{0}\right)=0 \quad \text { and } \quad h_{\mathbf{w}}\left(M_{\mathbf{v}: 1}\right)= \begin{cases}1, & \text { if } \mathbf{v}=\mathbf{w} \\ 0, & \text { if } \mathbf{v} \neq \mathbf{w}\end{cases}
$$

and for other neighborhood configurations $N \in \mathcal{N}$ by

$$
h_{\mathbf{w}}(N)=\sum_{\mathbf{v} \in V} h_{\mathbf{w}}\left(M_{\mathbf{v}: N(\mathbf{v})}\right)
$$

Each such local function $h_{\mathrm{w}}$ is called a split function.

The set of all split functions is denoted by $\mathcal{S}$, i.e.,

$$
\mathcal{S}=\left\{h_{\mathbf{0}}, h_{\mathbf{v}_{1}}, h_{-\mathbf{v}_{1}}, h_{\mathbf{v}_{2}}, h_{-\mathbf{v}_{2}}, \ldots, h_{\mathbf{v}_{d}}, h_{-\mathbf{v}_{d}}\right\} .
$$

As can be seen, a split function is defined by the values it takes on monomers, because its value on any neighborhood configuration is given by Eq. (5). For example, if $d=2$, then Eq. (5) means that for any $q_{1}, q_{2}, q_{3}, q_{4}, q_{5} \in Q$, it holds that

$$
\begin{aligned}
h_{\mathbf{w}}\left(\begin{array}{ccc}
q_{1} & \\
q_{2} & q_{3} & q_{4} \\
& q_{5} &
\end{array}\right)= & h_{\mathbf{w}}\left(\begin{array}{ccc}
q_{1} & \\
0 & 0 & 0 \\
0 &
\end{array}\right)+h_{\mathbf{w}}\left(\begin{array}{ccc} 
& 0 & \\
q_{2} & 0 & 0 \\
& 0 &
\end{array}\right) \\
& +h_{\mathbf{w}}\left(\begin{array}{ccc} 
& 0 & \\
0 & q_{3} & 0 \\
0 & 0
\end{array}\right)+h_{\mathbf{w}}\left(\begin{array}{ccc}
0 & 0 & q_{4} \\
0 & 0 &
\end{array}\right) \\
& +h_{\mathbf{w}}\left(\begin{array}{ccc}
0 & 0 & \\
0 & 0 & 0 \\
& q_{5} &
\end{array}\right) .
\end{aligned}
$$

Thus, for example, $h_{\mathbf{v}_{1}}\left(\begin{array}{c}q_{2} q_{1} \\ q_{3} \\ q_{5}\end{array} q_{4}\right)=h_{\mathbf{v}_{1}}\left(\begin{array}{cc}0 & 0 \\ 0 & q_{4}\end{array}\right)$.

Note that if $h \in \mathcal{S}$, then for any $\{\mathbf{u}, \mathbf{w}\} \in \boldsymbol{\Omega}$ and $p, q \in$ $\{0,1\}$, it holds that

$$
h\left(D_{\mathbf{w}: p}\right)=h\left(M_{\mathbf{u}: p}\right)+h\left(M_{\mathbf{w}: q}\right) .
$$

Each split function is number conserving (see Ref. [15]). Moreover, in the case of $Q=\{0,1\}$ all split functions are local rules, as $h_{\mathbf{v}}$ is simply the shift rule in direction $-\mathbf{v}$, if $\mathbf{v} \in V_{+}$, or the identity rule, if $\mathbf{v}=\mathbf{0}$. Thus, for given $d$, we have at least $2 d+1$ number-conserving local rules: the elements of $\mathcal{S}$.

Now we introduce the definition of a perturbation. Similarly, as in the case of a split function, we use the fact that $Q=\{0,1\}$ to present a simpler version of the definitions and facts given in Ref. [15].

Definition II.2. Let the set $\boldsymbol{\Lambda}$ be chosen. For each $\left\{\mathbf{u}_{0}, \mathbf{w}_{0}\right\} \in \boldsymbol{\Lambda}$ we define a basic perturbation $g_{\mathbf{u}_{0}, \mathbf{w}_{0}}$ as the local function that has the value 1 on the dimer $D_{\mathbf{u}_{0}: 1}$, the value 0 on the dimers defined by other pairs from $\boldsymbol{\Lambda}$ and on all monomers, while on other neighborhood configurations $N \in \mathcal{N}, g_{\mathbf{u}_{0}, \mathbf{w}_{0}}(N)$ equals

$$
\sum_{\{\mathbf{u}, \mathbf{w}\} \in \mathbf{\Lambda}}\left\{g_{\mathbf{u}_{0}, \mathbf{w}_{0}}\left(D_{\underset{\mathbf{w}}{\mathbf{u}}: N(\mathbf{w})}\right)-g_{\mathbf{u}_{0}, \mathbf{w}_{0}}\left(D_{\mathbf{w}: N(-\mathbf{u})}\right)\right\} .
$$

Let $\mathcal{P}$ be the linear space generated by

$$
\left\{g_{\mathbf{u}_{0}, \mathbf{w}_{0}} \mid\left\{\mathbf{u}_{0}, \mathbf{w}_{0}\right\} \in \boldsymbol{\Lambda}\right\} .
$$

The elements of $\mathcal{P}$ are called perturbations.
As can be seen, a perturbation is defined by the values it takes on dimers, because its value on any neighborhood configuration is given by Eq. (7). For example, if $d=2$ and we chose $\boldsymbol{\Lambda}$ as in Eq. (4), then Eq. (7) means that for any perturbation $g$ and any $q_{1}, q_{2}, q_{3}, q_{4}, q_{5} \in Q$, it holds that

$$
\begin{aligned}
g\left(\begin{array}{ccc}
q_{1} & \\
q_{2} & q_{3} & q_{4} \\
& q_{5} &
\end{array}\right)= & g\left(\begin{array}{ccc}
0 & \\
0 & q_{3} & q_{4} \\
0 &
\end{array}\right)-g\left(\begin{array}{ccc}
0 & \\
0 & q_{2} & q_{3} \\
0 &
\end{array}\right) \\
& +g\left(\begin{array}{ccc}
q_{1} & \\
0 & q_{3} & 0 \\
& 0 &
\end{array}\right)-g\left(\begin{array}{ccc}
q_{3} & \\
0 & q_{5} & 0 \\
0 &
\end{array}\right) \\
& +g\left(\begin{array}{cccc}
q_{1} & \\
0 & 0 & q_{4} \\
& 0 &
\end{array}\right)-g\left(\begin{array}{ccc}
q_{2} & \\
0 & 0 & q_{5} \\
0 &
\end{array}\right) \\
& +g\left(\begin{array}{cccc}
0 & 0 & \\
0 & 0 & q_{4}
\end{array}\right)-g\left(\begin{array}{ccc}
0 & 0 & \\
0 & 0 & q_{1} \\
& q_{5} &
\end{array}\right) .
\end{aligned}
$$

The next lemma shows the relationship between the values of a perturbation on matching dimers, proved in Ref. [15].

Lemma II.1. If a local function $g: \mathcal{N} \rightarrow \mathbb{R}$ is a perturbation, then for any $\{\mathbf{u}, \mathbf{w}\} \in \boldsymbol{\Omega}$ and any $p, q \in\{0,1\}$, it holds that

$$
g\left(D_{\mathbf{u}: p}\right)=-g\left(D_{-\mathbf{w}: p}\right)
$$

The main result of Ref. [15], reformulated in the case of $Q=\{0,1\}$, is presented in the following theorem.

Theorem II.1. Let the set $\boldsymbol{\Lambda}$ be chosen. The local rule $f$ of a binary CA with the von Neumann neighborhood is numberconserving if and only if it has the form

$$
f=h_{\mathbf{w}}+\sum_{\{\mathbf{u}, \mathbf{v}\} \in \mathbf{\Lambda}} a_{\mathbf{u}, \mathbf{v}} \cdot g_{\mathbf{u}, \mathbf{v}}
$$

where $\mathbf{w} \in V$ and the coefficients $a_{\mathbf{u}, \mathbf{v}}$ are chosen such that the values of $f$ are in $\{0,1\}$.

\section{ENUMERATION OF ALL $d$-DIMENSIONAL BINARY NCCAS}

Here we prove that all $d$-dimensional binary NCCAs with the von Neumann neighborhood act as a one-dimensional one. In contrast to the usual approaches in the field of CAs, our proof is very simple and does not require any heavy computational explorations. This is because the whole difficulty of the proof is hidden in the decomposition theorem (Theorem II.1). The remaining part of the argumentation is elementary and just a few lines long, as one can see below.

Let us note that for any $\mathbf{w} \in V$ there is at least one possibility of choosing the coefficients $a_{\mathbf{u}, \mathbf{v}}$ such that the local function $f$ given by Eq. (8) takes values in $\{0,1\}$. Indeed, we can put $a_{\mathbf{u}, \mathbf{v}}=0$, for all $\{\mathbf{u}, \mathbf{v}\} \in \mathbf{\Lambda}$, since then $f=h_{\mathbf{w}}$ and we know that each split function is a local rule (in the binary case). The question is whether there is any other possibility of choosing the coefficients $a_{\mathbf{u}, \mathbf{v}}$, i.e., whether we can perturb the split function $h_{\mathbf{w}}$ to get another local rule. 
First, we focus on the split function $h_{\mathbf{0}}$ and we show that there is no possibility to perturb this number-conserving local rule to get another one.

Lemma III.1. If a local function

$$
f=h_{\mathbf{0}}+\sum_{\{\mathbf{u}, \mathbf{v}\} \in \mathbf{\Lambda}} a_{\mathbf{u}, \mathbf{v}} \cdot g_{\mathbf{u}, \mathbf{v}}
$$

takes values in $\{0,1\}$, then all coefficients $a_{\mathbf{u}, \mathbf{v}}$ are equal to zero.

Proof. Let us consider some pair $\left\{\mathbf{u}_{0}, \mathbf{v}_{0}\right\} \in \boldsymbol{\Lambda}$. From Definition II.2 and Lemma II.1, it follows that $g_{\mathbf{u}_{0}, \mathbf{v}_{0}}\left(D_{\mathbf{u}_{\mathbf{v}_{0}}: 1}\right)=$ 1 and $g_{\mathbf{u}_{0}, \mathbf{v}_{0}}\left(D_{-\mathbf{u}_{0}: 1}\right)=-1$. Moreover, for any other pair $\{\mathbf{u}, \mathbf{v}\} \in \boldsymbol{\Lambda}$ we have $g_{\mathbf{u}, \mathbf{v}}\left(D_{\mathbf{u}_{0} \vdots 1}\right)=0$ and $g_{\mathbf{u}, \mathbf{v}}\left(D_{-\mathbf{v}_{0}: 1}\right)=0$. Hence,

$$
f\left(D_{\mathbf{u}_{0}: 1}\right)=h_{\mathbf{0}}\left(M_{\mathbf{u}_{0}: 1}\right)+h_{\mathbf{0}}\left(M_{\mathbf{v}_{0}: 1}\right)+a_{\mathbf{u}_{0}, \mathbf{v}_{0}}
$$

and

$$
f\left(D_{-\mathbf{u}_{0}} \vdots 1,1\right)=h_{\mathbf{0}}\left(M_{-\mathbf{u}_{0}: 1}\right)+h_{\mathbf{0}}\left(M_{-\mathbf{v}_{0}: 1}\right)-a_{\mathbf{u}_{0}, \mathbf{v}_{0}},
$$

according to Eq. (6). Note that $h_{\mathbf{0}}\left(M_{\mathbf{v}: 1}\right) \neq 0$ only if $\mathbf{v}=\mathbf{0}$. So, if the pair $\left\{\mathbf{u}_{0}, \mathbf{v}_{0}\right\}$ does not contain $\mathbf{0}$, then

$$
f\left(D_{\mathbf{u}_{0} \vdots 1}\right)=a_{\mathbf{u}_{0}, \mathbf{v}_{0}} \quad \text { and } \quad f\left(D_{-\mathbf{u}_{0} \vdots 1}\right)=-a_{\mathbf{u}_{0}, \mathbf{v}_{0}}
$$

otherwise, i.e., when one of $\mathbf{u}_{0}, \mathbf{v}_{0}$ equals $\mathbf{0}$, then

$$
f\left(D_{\mathbf{u}_{0} \vdots 1}\right)=1+a_{\mathbf{u}_{0}, \mathbf{v}_{0}} \quad \text { and } \quad f\left(D_{-\mathbf{u}_{0} \vdots 1}\right)=1-a_{\mathbf{u}_{0}, \mathbf{v}_{0}} .
$$

As the values of $f$ have to belong to $\{0,1\}$, in both cases $a_{\mathbf{u}_{0}, \mathbf{v}_{0}}$ needs to be zero. This finishes the proof.

Now we consider the split function $h_{\mathbf{w}}$, where $\mathbf{w} \in V_{+}$. This case is more complicated: There is exactly one possibility to perturb the number-conserving local rule $h_{\mathbf{w}}$ to get another one. As the proof is the same for any direction $\mathbf{w} \in V_{+}$, we will deal with $\mathbf{v}_{1}$ only. To simplify our reasoning, let us choose $\boldsymbol{\Lambda}$ given in Eq. (3). Then it holds that $\left\{\mathbf{0}, \mathbf{v}_{1}\right\} \in \boldsymbol{\Lambda}$, leading to the following result.

Lemma III.2. If a local function

$$
f=h_{\mathbf{v}_{1}}+\sum_{\{\mathbf{u}, \mathbf{v}\} \in \mathbf{\Lambda}} a_{\mathbf{u}, \mathbf{v}} \cdot g_{\mathbf{u}, \mathbf{v}}
$$

takes values in $\{0,1\}$, then either all coefficients $a_{\mathbf{u}, \mathbf{v}}$ are equal to zero or $a_{\mathbf{0}, \mathbf{v}_{1}}=-1$ and all other coefficients $a_{\mathbf{u}, \mathbf{v}}$ are equal to zero.

Proof. First, let us consider a pair $\left\{\mathbf{u}_{0}, \mathbf{w}_{0}\right\} \in \boldsymbol{\Lambda}$ such that $\mathbf{u}_{0}, \mathbf{w}_{0} \in V \backslash\left\{\mathbf{v}_{1},-\mathbf{v}_{1}\right\}$. According to Eq. (6) and Definition II.2, we have

$$
f\left(D_{\mathbf{u}_{0}: 1,1}\right)=a_{\mathbf{u}_{0}, \mathbf{v}_{0}} \quad \text { and } \quad f\left(D_{-\mathbf{u}_{0}: 1}\right)=-a_{\mathbf{u}_{0}, \mathbf{v}_{0}},
$$

which implies $a_{\mathbf{u}_{0}, \mathbf{v}_{0}}=0$.

Next, let us consider any $\mathbf{v}_{k} \in\left\{\mathbf{v}_{2}, \mathbf{v}_{3}, \ldots, \mathbf{v}_{d}\right\}$. As $\left\{\mathbf{v}_{1}, \mathbf{v}_{k}\right\},\left\{-\mathbf{v}_{1}, \mathbf{v}_{k}\right\} \in \boldsymbol{\Lambda}$ [see Eq. (3)] it holds that

$$
\begin{array}{r}
f\left(D_{\mathbf{v}_{k}: 1}\right)=1+a_{\mathbf{v}_{1}, \mathbf{v}_{k}}, \quad f\left(D_{-\mathbf{v}_{1}: 1}\right)=a_{-\mathbf{v}_{1}, \mathbf{v}_{k}}, \\
f\left(D_{\substack{\mathbf{v}_{1}: 1 \\
-\mathbf{v}_{k}: 1}}\right)=1-a_{-\mathbf{v}_{1}, \mathbf{v}_{k}}, \quad f\left(D_{-\mathbf{v}_{1}: 1}\right)=-a_{\mathbf{v}_{1}, \mathbf{v}_{k}} .
\end{array}
$$

Moreover, for three neighborhood configurations $N_{1}, N_{2}, N_{3}$ defined by

$$
N_{i}(\mathbf{v})= \begin{cases}1, & \text { if } \mathbf{v} \in B_{i} \\ 0, & \text { otherwise }\end{cases}
$$

where $B_{1}=\left\{\mathbf{v}_{1},-\mathbf{v}_{1}, \mathbf{v}_{k}\right\}, B_{2}=\left\{\mathbf{v}_{1},-\mathbf{v}_{1},-\mathbf{v}_{k}\right\}$, and $B_{3}=$ $\left\{\mathbf{v}_{1}, \mathbf{v}_{k},-\mathbf{v}_{k}\right\}$, we have

$$
\begin{aligned}
& f\left(N_{1}\right)=1+a_{\mathbf{v}_{1}, \mathbf{v}_{k}}+a_{-\mathbf{v}_{1}, \mathbf{v}_{k}}, \\
& f\left(N_{2}\right)=1-a_{\mathbf{v}_{1}, \mathbf{v}_{k}}-a_{-\mathbf{v}_{1}, \mathbf{v}_{k}}, \\
& f\left(N_{3}\right)=1+a_{\mathbf{v}_{1}, \mathbf{v}_{k}}-a_{-\mathbf{v}_{1}, \mathbf{v}_{k}} .
\end{aligned}
$$

As all results obtained in Eqs. (9) and (10) have to belong to $\{0,1\}$, we get $a_{\mathbf{v}_{1}, \mathbf{v}_{k}}=a_{-\mathbf{v}_{1}, \mathbf{v}_{k}}=0$.

Finally, we consider the last pair $\left\{\mathbf{0}, \mathbf{v}_{1}\right\}$ from $\boldsymbol{\Lambda}$. Since

$$
f\left(D_{\mathbf{v}_{1}: 1}\right)=1+a_{\mathbf{0}, \mathbf{v}_{1}} \text { and } f\left(D_{-\mathbf{v}_{1}: 1}^{D_{\mathbf{0}}: 1}\right)=-a_{\mathbf{0}, \mathbf{v}_{1}},
$$

it follows that $a_{\mathbf{0}, \mathbf{v}_{1}}=0$ or $a_{\mathbf{0}, \mathbf{v}_{1}}=-1$. Let us note that both solutions yield a local rule. In the first case, all coefficients $a_{\mathbf{u}, \mathbf{v}}$ are equal to zero, thus $f=h_{\mathbf{v}_{1}}$, which means that $f$ is simply the shift rule along vector $-\mathbf{v}_{1}$. In the second case, $f=h_{\mathbf{v}_{1}}-g_{\mathbf{0}, \mathbf{v}_{1}}$, so $f$ is just the traffic rule in the direction $-\mathbf{v}_{1}$. The lemma is proved.

The considerations presented above result in the following theorem.

Theorem III.1. There are exactly $4 d+1$ numberconserving $d$-dimensional binary CAs with the von Neumann neighborhood: the identity rule and the shift and the traffic rules in each of the $2 d$ possible directions.

How does the dynamics of a $d$-dimensional binary NCCA with the von Neumann neighborhood look like? If we are dealing with a shift rule (say, in a direction w), then the initial pattern of a configuration does not change, it only moves in the direction $\mathbf{w}$ by one cell in each time step. So although Theorem III.1 states that such CA acts independently on every one-dimensional $\mathbf{w}$-row of the grid $\mathcal{C}$ (i.e., a row extending in the direction $\mathbf{w}$ ), in the case of a shift rule we observe a synchronous action as an aggregate result. The case of traffic rules is different. For example, the traffic rule in direction $\mathbf{v}_{1}$ acts as follows. The pattern of each $\mathbf{v}_{1}$-row quickly stabilizes. Subsequently, if in a given $\mathbf{v}_{1}$-row the number of $1 \mathrm{~s}$ is smaller than the number of $0 \mathrm{~s}$, then in every consecutive time step, the $1 \mathrm{~s}$ seem to go one step in the direction $\mathbf{v}_{1}$. If the number of $1 \mathrm{~s}$ is greater than the number of $0 \mathrm{~s}$, then in every consecutive time step, the 0 s seem to go one step in the direction $-\mathbf{v}_{1}$. If the number of $1 \mathrm{~s}$ equals the number of $0 \mathrm{~s}$, then the pattern stabilizes as an alternating sequence of 0 s and 1 s, which moves with time in the direction $\mathbf{v}_{1}$. A more detailed description of the dynamics of traffic rules can be found, for example, in Ref. [17].

\section{CONCLUDING REMARKS}

In this paper, we have considered $d$-dimensional binary number-conserving cellular automata with the von Neumann neighborhood and we have proved that for any given $d$ there are exactly $4 d+1$ such cellular automata: the identity rule and the shift and traffic rules in each of the $2 d$ possible directions. Thus all of them are in some sense trivial, since they act as one-dimensional ones. 
Let us note that if we would like to add one more assumption, namely reversibility (a widely accepted principle in physics), then only the identity rule and the shift rules remain, since the traffic rules are not reversible. What does this mean in the context of the modeling of particle systems? Suppose that we want to use cellular automata to model a system of interacting particles in a $d$-dimensional grid, and we assume that there is no force acting on the particles, but we want that the local law governing the dynamics can be reversed. If we use cellular automata with the von Neumann neighborhood, then we get only two possibilities for the local rule: the identity rule (the particles stay at rest) or the shift rule (the particles stay in motion along a straight line at a constant speed), which coincides with Newton's first law of motion.

One could ask what the minimal state set needed is to obtain a nontrivial reversible $d$-dimensional number-conserving cellular automaton. If $d=1$, then there are 144 ternary number-conserving CAs [18], but only three among them are reversible: the identity rule and two shift rules. Deciding reversibility of a $\mathrm{CA}$ in two or higher dimensions is considered to be a hard problem. It was shown to be an undecidable property in general [19]. Restrictions to finite grids are obviously decidable but still computationally hard (see, for example, Ref. [20]). In Ref. [21], all 1327 ternary two-dimensional number-conserving CAs are enumerated and it is proven that there is no nontrivial reversible one. We conjecture that the same holds true for higher dimensions, i.e., that three states are too few to allow for the existence of a reversible number-conserving cellular automaton and we believe that it is possible to prove this fact by using the decomposition theorem. We plan to investigate this problem in the near future.

Another direction of future research is to build similar mathematical tools for the study of NCCAs in other settings. The basic idea of the split-and-perturb decomposition of the local rule of an NCCA, presented here for the von Neumann neighborhood, can also be considered for the Moore neighborhood. The difficulty then, however, lies in describing the space of perturbations. At this moment, we do not have any intuition about how rich the set of all $d$-dimensional binary NCCAs with the Moore neighborhood could be.
[1] S. Ulam, in Proceedings of Symposia in Applied Mathematics, Vol. 14 (American Mathematical Society, Providence, RI, 1962), pp. 215-224.

[2] J. von Neumann, in Theory of Self-Reproducing Automata, edited by A. W. Burks (University of Illinois Press, Champaign, IL, 1966).

[3] R. Bowness, M. A. Chaplain, G. G. Powathil, and S. H. Gillespie, J. Theor. Biol. 446, 87 (2018).

[4] T. Nagatani and Kei-ichi Tainaka, Physica A 490, 803 (2018).

[5] M. Bakhtiari and M. S. Salehi, Comput. Mater. Sci. 149, 1 (2018).

[6] Z. Fu, Q. Jia, J. Chen, J. Ma, K. Han, and L. Luo, Transport. Res. C 91, 37 (2018).

[7] S. Iwan, K. Kijewska, B. G. Johansen, O. Eidhammer, K. Małecki, W. Konicki, and R. G. Thompson, Transport. Res. D 61, 104 (2018).

[8] D. Caviedes-Voullième, J. Fernández-Pato, and C. Hinz, J. Hydrol. 563, 411 (2018).

[9] R. Zhang, Q. Tian, L. Jiang, A. Crooks, S. Qi, and R. Yang, Land Use Policy 74, 248 (2018).
[10] M. Medenjak, K. Klobas, and T. Prosen, Phys. Rev. Lett. 119, 110603 (2017).

[11] G. 't Hooft, The Cellular Automaton Interpretation of Quantum Mechanics, Fundamental Theories of Physics, Vol. 185 (Springer International Publishing, Cham, 2016).

[12] B. Durand, E. Formenti, and Z. Róka, Theor. Comput. Sci. 299, 523 (2003).

[13] S. Wolfram, Rev. Mod. Phys. 55, 601 (1983).

[14] B. Wolnik, A. Dzedzej, J. M. Baetens, and B. De Baets, J. Phys. A: Math. Theor. 50 (2017).

[15] B. Wolnik, A. Nenca, J. M. Baetens, and B. De Baets, A split-and-perturb decomposition of number-conserving cellular automata, arXiv:1901.05067.

[16] M. Land and R. K. Belew, Phys. Rev. Lett. 74, 5148 (1995).

[17] H. Fukś, Phys. Rev. E 55, R2081 (1997).

[18] N. Boccara and H. Fukś, Fundam. Inf. 52, 1 (2002).

[19] J. Kari, Physica D 45, 379 (1990).

[20] B. Durand, Theor. Comput. Sci. 134, 387 (1994).

[21] A. Dzedzej, B. Wolnik, A. Nenca, J. M. Baetens, and B. De Baets, Efficient enumeration of three-state two-dimensional number-conserving cellular automata (unpublished). 